\title{
Targeted therapies in the management of colorectal carcinoma: role of bevacizumab
}

\author{
Ajithkumar Puthillath' \\ Anush Patel ${ }^{2}$ \\ Marwan G Fakih' \\ 'Department of Medicine at Roswell \\ Park Cancer Institute, Buffalo, New \\ York, USA; ${ }^{2}$ Department of Medicine \\ at State University of Buffalo, Buffalo, \\ New York, USA
}

\begin{abstract}
Colorectal cancer continues to be an important public health concern, despite improvements in screening and better systemic chemotherapy. The integration of targeted therapies in the treatment of colon cancer has resulted in significant improvements in efficacy outcomes. Angiogenesis is important for tumor growth and metastasis and is an important target for new biological agents. Bevacizumab is a humanized recombinant antibody that prevents vascular endothelial growth factor (VEGF) receptor binding, and inhibits angiogenesis and tumor growth. The addition of bevacizumab to fluoropyrimidine-based chemotherapy, with or without irinotecan or oxaliplatin, in both the first- and second-line treatment of metastatic colorectal cancer, significantly increased median progression-free survival and overall survival in select randomized phase III studies. Ongoing studies are evaluating the role of bevacizumab in the adjuvant treatment of colon cancer. Common toxicities associated with bevacizumab include hypertension, bleeding episodes, and thrombotic events. This review will focus on the integration of bevacizumab in the treatment paradigm of colon cancer and the management of its side effects.
\end{abstract}

Keywords: colorectal cancer, metastatic, bevacizumab, fluorouracil, irinotecan, oxaliplatin

\section{Introduction}

Colorectal cancer (CRC) is the second leading cause of death in the US. An estimated 148,810 cases of CRC are expected to occur in 2008, resulting in 49,960 deaths, almost $10 \%$ of all cancer deaths. ${ }^{1}$ Overall, the 5 -year survival for all patients has improved significantly from $41 \%$ in $1950-54$ to $66 \%$ in $1996-2004 .{ }^{2}$ Survival is still dependent on stage of disease, with the 5-year survival ranging from $85 \%$ to $90 \%$ in stage I disease to $\approx 10 \%$ for patients with stage IV disease. . $^{3,4}$

For several decades 5-fluorouracil (FU)/leucovorin (LV)-based therapy was the mainstay of treatment of CRC. In the past decade, the outcome of patients with metastatic $\mathrm{CRC}$ has improved considerably with the advent of combination regimens of oxaliplatin or irinotecan and 5-FU/LV.,6 The addition of irinotecan to a bolus or infusional regimen of 5-FU in combination with $\mathrm{LV}$ in the first line setting has resulted in a median survival of 15 to 23 months. ${ }^{6-8}$ Infusional 5-FU/LV is clearly less toxic and slightly more efficacious than bolus administration and hence has evolved to become the preferred regimen in combination with irinotecan (FOLFIRI) and oxaliplatin (FOLFOX). Most recently, the incorporation of monoclonal antibodies that bind to vascular endothelial growth factor (VEGF) and to epidermal growth factor receptors (EGFR) into the current armamentarium has further widened the treatment options..$^{9,10}$ Irrespective of the first-line chemotherapy regimen used, when patients are exposed to all active cytotoxic drugs available against CRC an overall survival (OS) exceeding 2 years is currently achieved. ${ }^{11}$ In this review we will discuss the role of bevacizumab in the treatment of CRC and briefly discuss its side effect profile. 


\section{VEGF pathway}

Angiogenesis is a complex multistep process of new blood vessel formation and is considered critical for the growth of tumors. ${ }^{12}$ In 1971, Judah Folkman first proposed that tumor angiogenesis could serve as a potential target for anticancer therapy. ${ }^{13}$ One of the major pathways involved in this process is the VEGF family of proteins and receptors. ${ }^{14,15}$ VEGF is a diffusible homodimeric glycoprotein produced by healthy and neoplastic cells and is a key promoter of angiogenesis under both physiological and pathological conditions, including tumor progression. ${ }^{16}$

The VEGF family includes 6 members referred to as VEGF-A through VEGF-E and placental growth factor (PIGF). It is recognized that the major mediator of tumor angiogenesis is VEGF-A (referred to as VEGF henceforth in this review). ${ }^{17-19}$ VEGF ligands mediate their angiogenic effects mainly through 3 different cell membrane receptors, VEGF R-1, -2 and $-3 .{ }^{20-25}$ These receptors consist of an extracellular domain, a transmembrane domain and an intracellular tyrosine kinase domain. Binding of the ligand to the receptor induces the activation of intracellular signaling transduction pathways that are involved in the regulation of cellular proliferation and survival, such as the raf/MEK, mTOR and PI3K pathways (see Figure 1). The neuropilins are a class of transmembrane proteins (NRP-1 and NRP-2) that lack tyrosine kinase activity but act as co receptors for the VEGF receptors, increasing their binding affinity. ${ }^{26-29}$ Most recently, alternative signaling pathways (notch-delta like ligand) have been studied and appear to be important regulators in the angiogenesis pathway.

The expression of VEGF is increased in most human tumors (including CRC), with contributions from both malignant and host cells. Several studies have shown that VEGF levels correlate with increased micro vessel density, higher incidence of metastasis, decreased apoptotic index and poor OS of patients with CRC. ${ }^{30-34}$ Because VEGF and its receptors play a critical role in angiogenesis and tumor progression, initial work focused on the development of agents that specifically inhibit this pathway. Several preclinical models demonstrated that monoclonal antibodies directed against VEGF inhibit tumor growth in mice. ${ }^{35,36}$ In an experimental liver metastasis model, blocking VEGF with murine monoclonal antibody (A.4.6.1) showed reduction in the size and the number of liver metastasis in mice. ${ }^{37}$ Preclinical models also showed a synergistic effect combining anti-VEGF antibodies with cytotoxic chemotherapy or radiation therapy. ${ }^{38-40}$
Bevacizumab is a recombinant, humanized, monoclonal antibody directed against the VEGF ligand (VEGF-A) and binds all isoforms of VEGF-A with high affinity. In the initial phase 1 study of bevacizumab in patients with advanced cancer, bevacizumab was safely administered without doselimiting toxicity at doses ranging up to $10 \mathrm{mg} / \mathrm{kg}{ }^{41,42}$ Bevacizumab was well tolerated, and pharmacokinetic studies indicated that doses of $\geq 0.3 \mathrm{mg} / \mathrm{kg}$ had a half-life similar to that of other humanized antibodies. Bevacizumab showed a linear pharmacokinetic profile and a terminal half-life of around 21 days. Subsequently several phase II studies were initiated with bevacizumab single agent or in combination with chemotherapy in the treatment of various solid tumors with promising results. ${ }^{43-46}$

\section{Bevacizumab in the first-line treatment of metastatic CRC}

In colorectal cancer, two dosages of bevacizumab have been investigated after a randomized phase II study of high and low dose bevacizumab in combination with 5-FU/LV showed considerable improvement in efficacy when compared to the 5 -FU/LV control. ${ }^{47}$ One hundred and four previously untreated patients with measurable metastatic CRC were randomly assigned to one of three treatment groups: 5-FU $\left(500 \mathrm{mg} / \mathrm{m}^{2}\right)$ and $\mathrm{LV}\left(500 \mathrm{mg} / \mathrm{m}^{2}\right)$ (weekly for 6 weeks of each 8-week cycle), 5-FU/LV plus bevacizumab ( $5 \mathrm{mg} / \mathrm{kg}$ once every 2 weeks), and 5-FU/LV plus bevacizumab $(10 \mathrm{mg} / \mathrm{kg}$ once every 2 weeks). Response rates were higher in both bevacizumab arms: $17 \%$ for FU/LV alone, $40 \%$ for FU/LV/low-dose bevacizumab and $24 \%$ for FU/LV/ highdose bevacizumab. Furthermore, time to disease progression was longer in both bevacizumab arms: 5.2 months for FU/LV alone, 9.0 months for FU/LV/low-dose bevacizumab, and 7.2 months with FU/LV/high-dose bevacizumab with a trend towards improved survival (Table 1). The major safety concerns were gastrointestinal (GI) bleeding (0, 6\% and 16\%) and thrombosis $(9 \%, 26 \%$ and $13 \%)$ seen in the $5-\mathrm{FU} / \mathrm{LV}$, $5-\mathrm{FU} / \mathrm{LV}$ with $5 \mathrm{mg} / \mathrm{kg}$ bevacizumab and 5-FU/LV with $10 \mathrm{mg} / \mathrm{kg}$ bevacizumab, respectively.

\section{Bevacizumab in combination with irinotecan-based regimens}

The clinical benefit seen with the addition of bevacizumab to $5-\mathrm{FU} / \mathrm{LV}$ based chemotherapy in phase II studies was further studied in a pivotal phase 3 study. ${ }^{9}$ Patients were initially randomized in a 1:1:1 ratio to 6 -week cycles of IFL (irinotecan $125 \mathrm{mg} / \mathrm{m}^{2}$, 5 -FU $500 \mathrm{mg} / \mathrm{m}^{2}$ and $\mathrm{LV}$ $20 \mathrm{mg} / \mathrm{m}^{2}$ once a week for 4 weeks plus bevacizumab 


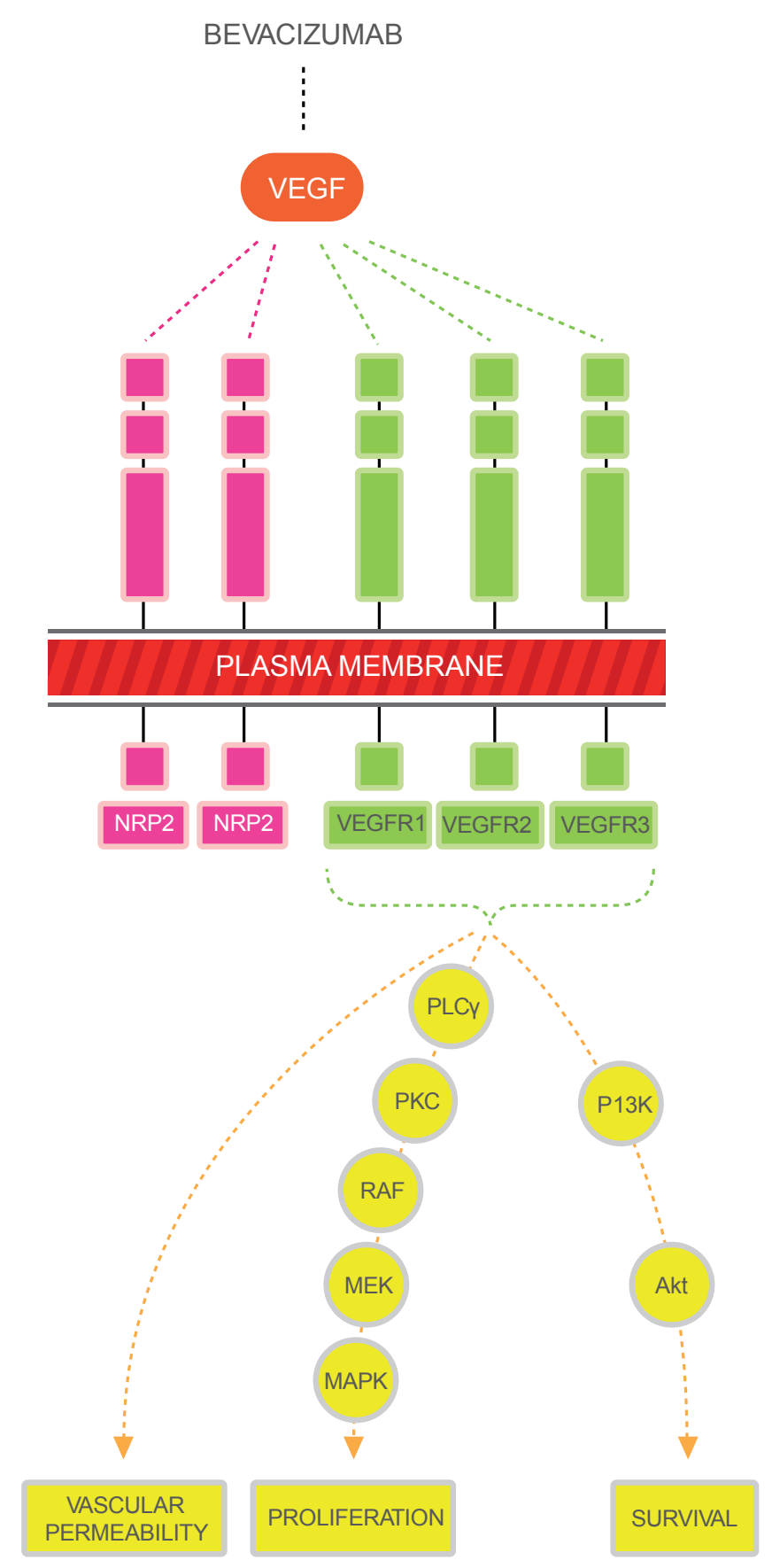

Figure I The major mediator of tumor angiogenesis is the vascular endothelial growth factor (VEGF) family of growth factors. The VEGF family binds to the VEGF receptors (VEGFR)-RI,VEGFR-2,VEGFR-3 tyrosine kinase as shown in figure. Neuropilin (NRP I and 2) are co-receptors for the VEGFRs and lack tyrosine kinase activity. The binding ofVEGF to theVEGF receptors results in activation of the intracellular pathways. Activation of the PLC-PKC-raf kinase-MEK-mitogen activated protein kinase pathway results in increased cell proliferation. Activation of the phosphatidylinositol 3'-kinase (PI3K), akt pathway leads to increased cell survival.

$5 \mathrm{mg} / \mathrm{kg}$ every 2 weeks), 6-week cycles of IFL plus placebo, or 8 -week cycles of 5 -FU $\left(500 \mathrm{mg} / \mathrm{m}^{2}\right)$ and $\mathrm{LV}\left(500 \mathrm{mg} / \mathrm{m}^{2}\right)$ once a week for 6 weeks) plus bevacizumab $(5 \mathrm{mg} / \mathrm{kg})$. After 300 patients were randomized, an unblinded safety analysis found that the IFL plus bevacizumab regimen had an acceptable safety profile, and randomization to the 5-FU/LV plus bevacizumab arm was discontinued as per the pre-planned design. Compared with IFL alone, IFL plus bevacizumab improved the progression-free survival (PFS) from a median of 6.2 months to 10.6 months, the overall response rate from $34.8 \%$ to $44.8 \%$, and the median OS from 15.6 to 20.3 months. The clinical benefit of bevacizumab was seen across all pre-specified patient subgroups including age, performance status, location and number of metastatic sites. 
Table I Initial phase II trials of bevacizumab in metastatic colorectal cancer

\begin{tabular}{llllll}
\hline Phase II studies & Chemotherapy regimen & $\mathbf{n}$ & $\begin{array}{l}\text { Time to progression } \\
\text { (months) }\end{array}$ & Response rate & $\begin{array}{l}\text { Median survival } \\
\text { (months) }\end{array}$ \\
\hline Kabbinavar et al $^{47}$ & $5-\mathrm{FU} / \mathrm{LV}$ & 36 & 5.2 & $6(17 \%)$ & 13.8 \\
& $5-\mathrm{FU} / \mathrm{LV}+\mathrm{BV} 5 \mathrm{mg} / \mathrm{kg}$ & 35 & 9 & $14(40 \%)$ & 21.5 \\
& $5-\mathrm{FU} / \mathrm{LV}+\mathrm{BV} 10 \mathrm{mg} / \mathrm{kg}$ & 33 & 7.2 & $8(24 \%)$ & 16.1 \\
Kabbinavar et al $^{57}$ & $5-\mathrm{FU} / \mathrm{LV}$ & 105 & 5.5 & $15.2 \%$ & 12.9 \\
& $5-\mathrm{FU} / \mathrm{LV}+\mathrm{BV} 5 \mathrm{mg} / \mathrm{kg}$ & 104 & 9.2 & $26 \%$ & 16.6 \\
\hline
\end{tabular}

Abbreviation: BV, bevacizumab.

On February 26, 2004 the FDA approved bevacizumab as a first-line treatment in metastatic CRC.

Given the synergistic activity of bevacizumab when added to IFL or to 5-FU/LV, bevacizumab was incorporated into different schedules of irinotecan-based regimens (Table 2). The combination of bevacizumab and FOLFIRI was studied in two small phase II studies with good tolerability and efficacy. ${ }^{48,49}$ These results were further explored in a number of phase III studies.

In the complex BICC-C trial, patients with previously untreated metastatic $\mathrm{CRC}$ were randomized to: infusional 5-FU/LV with irinotecan, modified IFL (mIFL: bolus 5 -FU/LV/irinotecan given weekly $\times 2$ every 3 weeks), or capecitabine with irinotecan. Patients were randomized further to receive concurrent celecoxib or placebo in a doubleblind fashion. Upon approval of bevacizumab in the first-line treatment of metastatic CRC in the US, the protocol was amended to add bevacizumab and to limit the randomization to FOLFIRI and mIFL arms with or without celecoxib. Study data presented in ASCO 2007 showed the bevacizumab plus FOLFIRI arm with a median PFS of 11.2 months, 1-year survival of $87 \%$ and a median OS of 28 months. The median OS of FOLFIRI plus bevacizumab compared favorably to mIFL plus bevacizumab (median, 19.2 months; $\mathrm{p}=0.037$ ).
However, the data are limited by the small sample size (57 patients treated with FOLFIRI plus bevacizumab). ${ }^{50,51}$ A phase IV study of first-line bevacizumab plus FOLFIRI in patients with metastatic CRC (AVIRI study) lends further support to the integration of bevacizumab with FOLFIRI in the first-line setting. ${ }^{52} \mathrm{~A}$ total of 209 patients were enrolled at 31 centers worldwide. Interim efficacy results showed a median PFS of 11.1 months and a response rate (RR) of $53.1 \%$. Median OS data were not available at the time of this report.

\section{Bevacizumab in combination with oxaliplatin-based regimens}

The landmark N 9741 study compared oxaliplatin plus infusional 5-FU/LV (FOLFOX) regimen to IFL and to IROX (irinotecan plus oxaliplatin combination). This study showed a statistically significant improvement in time to progression (TTP) and OS for FOLFOX (oxaliplatin $85 \mathrm{mg} / \mathrm{m}^{2}$ on day 1 and bolus FU $400 \mathrm{mg} / \mathrm{m}^{2}$ plus LV $200 \mathrm{mg} / \mathrm{m}^{2}$ followed by FU $600 \mathrm{mg} / \mathrm{m}^{2}$ in 22-hour infusions on days 1 and 2 every 2 weeks) over IFL (irinotecan $125 \mathrm{mg} / \mathrm{m}^{2}$ and bolus FU $500 \mathrm{mg} / \mathrm{m}^{2}$ plus LV $20 \mathrm{mg} / \mathrm{m}^{2}$ on days 1,8 , 15 and 22 every 6 weeks ) and IROX (oxaliplatin $85 \mathrm{mg} / \mathrm{m}^{2}$ and irinotecan $200 \mathrm{mg} / \mathrm{m}^{2}$ every 3 weeks) resulting in the

Table 2 Selected randomized studies of bevacizumab in first-line metastatic colorectal cancer

\begin{tabular}{|c|c|c|c|c|c|c|}
\hline Studies & Phase & Chemotherapy regimen & $\mathbf{n}$ & $\begin{array}{l}\text { Time to progression } \\
\text { (months) }\end{array}$ & Response rate (\%) & $\begin{array}{l}\text { Median survival } \\
\text { (months) }\end{array}$ \\
\hline Hurwitz ${ }^{9}$ & III & $\mathrm{IFL}+\mathrm{BV} 5 \mathrm{mg} / \mathrm{kg}$ & 402 & 10.6 & 44.8 & 20.3 \\
\hline \multirow[t]{2}{*}{ BICC-C ${ }^{8}$} & III & FOLFIRI + BV $5 \mathrm{mg} / \mathrm{kg}$ & 57 & 11.2 & 57.9 & 28 \\
\hline & & $\mathrm{mlFL}+\mathrm{BV} 5 \mathrm{mg} / \mathrm{kg}$ & 60 & 8.3 & 53.3 & 19.2 \\
\hline$A V I R I^{52}$ & IV & FOLFIRI + BV $5 \mathrm{mg} / \mathrm{kg}$ & 209 & II.I & 44 & $N^{a}$ \\
\hline \multirow[t]{3}{*}{ TREE- $2^{54}$} & II & FOLFOX + BV 5 mg/kg & 71 & 9.9 & 52 & 26.1 \\
\hline & & $\mathrm{bFOL}+\mathrm{BV} 5 \mathrm{mg} / \mathrm{kg}$ & 70 & 8.3 & 39 & 20.4 \\
\hline & & CapeOX + BV 5 mg/kg & 72 & 10.3 & 46 & 24.6 \\
\hline NI6966 55 & III & FOLFOX or XELOX + BV $5 \mathrm{mg} / \mathrm{kg}$ & 699 & 9.4 & 49 & 21.3 \\
\hline
\end{tabular}

${ }^{\mathrm{a} N R}$, not reported. 
approval of FOLFOX chemotherapy in the first-line treatment of MCRC. ${ }^{53}$ Subsequent studies investigated if the addition of bevacizumab to oxaliplatin-based regimens improve patient outcome. In the TREE-2 trial, 213 previously untreated patients with metastatic CRC were randomly assigned to bevacizumab ( $5 \mathrm{mg} / \mathrm{kg}$ every 2 weeks) and one of the three different oxaliplatin and 5-FU-containing regimens used in the TREE 1 trial - FOLFOX (mFOLFOX6 - bolus and infusion fluorouracil [FU] and leucovorin [LV] with oxaliplatin), oxaliplatin plus bolus 5-FU/LV (bFOL), or capecitabine plus oxaliplatin (CapeOx). ${ }^{54}$ While the bFOL arm was significantly less active than FOLFOX, CapeOx and FOLFOX showed similar activity. Bevacizumab significantly improved the response rates of all of the regimens. The bevacizumabcontaining arms, combined, resulted in a median OS of 23.7 months. This compared favorably with the median OS of 18.2 months for the combined non-bevacizumab-containing groups on the TREE-1 study.

However, a large randomized phase III trial, NO16966, failed to show an advantage for the addition of bevacizumab to oxaliplatin-based first-line treatment in term of RR or OS, although PFS was marginally improved. ${ }^{55}$ The study randomized 1,400 patients with metastatic CRC to receive first-line FOLFOX4 versus capecitabine plus oxaliplatin (XELOX), with further randomization to bevacizumab or placebo. Bevacizumab was administered at $5 \mathrm{mg} / \mathrm{kg}$ intravenously every 2 weeks on the FOLFOX regimen and at $7.5 \mathrm{mg} / \mathrm{kg}$ every 3 weeks on the XELOX regimen. Median PFS was 9.4 months in the bevacizumab arm compared with 8 months in the non-bevacizumab arm $(\mathrm{HR}=0.83, \mathrm{p}=0.0023)$. However, unlike prior phase III bevacizumab studies, no benefit was documented in OS (21.3 for the bevacizumab arm versus 19.9 months for the placebo arms) and RR (38\% on both arms) with the addition of bevacizumab., ${ }^{9,56}$ This study was plagued by a high rate of unjustified discontinuation of study treatment because of toxicity concerns despite lack of progressive disease. In fact, treatment was discontinued secondary to progressive disease only in $29 \%$ of patients with bevacizumab compared with $47 \%$ in the placebo groups. The duration of treatment with bevacizumab in the initial IFL with and without bevacizumab pivotal study was 2.8 months longer compared with NO16966, likely due to the lack of dose-limiting neuropathy with IFL treatment. ${ }^{9}$ When NO16966 was analyzed using the pre-specified secondary analysis of on-treatment PFS (patients who progressed or died within 28 days from the last dose of any component of study treatment), the median on treatment PFS was 10.4 months versus 7.9 months, favoring the bevacizumab $\operatorname{arm}($ HR 0.63; 97.5\% CI, 0.52-0.75; p < 0.001). Despite the fact that early bevacizumab discontinuation may explain the limited benefit in terms of PFS and lack of OS advantage, it does not explain the lack of benefit in terms of RR, a finding consistently seen in previous studies with bevacizumab. ${ }^{9,54,56}$ In prior trials of bevacizumab in metastatic CRC in the firstline setting, RR increased by more than $\geq 10 \%$, in comparison with the non-bevacizumab arms. ${ }^{9,56,57}$ Similarly, the addition of bevacizumab in the second-line setting to FOLFOX-4 demonstrated a $13 \%$ improvement in RR compared with FOLFOX-4 alone.

Currently none of the phase III studies have evaluated the value of bevacizumab in combination with the stop-and-go approach used with oxaliplatin-based regimens. ${ }^{58}$ The CONcepT trial evaluated a standard FOLFOX (mFOLFOX7 - oxali $85 \mathrm{mg} / \mathrm{m}^{2}$, LV $200 \mathrm{mg} / \mathrm{m}^{2}$, 5FU $2,400 \mathrm{mg} / \mathrm{m}^{2} \times 46$ hours) plus bevacizumab regimen versus a stop-and-go FOLFOX plus bevacizumab approach with or without intravenous calcium /magnesium supplementation. ${ }^{59}$ The study was prematurely closed due to initial efficacy concerns in the calcium/magnesium arms. The final analysis of the initial cohort of patients $(n=140)$ showed a higher time to treatment failure (TTF) (5.6 versus 4.2 months) without compromising PFS (12 versus 7.3 months), favoring patients in the stop-and-go schedule with bevacizumab. These data support the feasibility of a stop-and-go approach with FOLFOX-based regimens and suggest the superiority of such an approach over the administration of FOLFOX until toxicity or progression when combined with bevacizumab.

\section{Bevacizumab plus FOLFIRI versus bevacizumab plus FOLFOX}

Currently there are no randomized phase III studies which compare FOLFOX plus bevacizumab versus FOLFIRI plus bevacizumab. Results from the First BEAT trial, a large international community-based prospective observational study which enrolled 1,965 patients to evaluate the safety and efficacy profile of bevacizumab when combined with a variety of first-line chemotherapy regimens in a general patient population with metastatic CRC, were presented at ASCO $2008 .{ }^{60}$ Most commonly used regimens in combination with bevacizumab included FOLFOX (29\%), FOLFIRI (26\%), XELOX (18\%) and 5-FU/capecitabine monotherapy (15\%). The overall median PFS was 10.8 months and did not show major differences in PFS between the different combination therapy regimens. Overall survival was 22.7 months, with no major differences between the FOLFOX and FOLFIRI regimens (25.9 versus 23.7 months). 
Currently the integration of bevacizumab in the first-line treatment of metastatic CRC irrespective of which first-line regimen is chosen should be strongly considered. This is now supported not only by prospective randomized studies but also by large observational studies showing PFS in excess of 10.5 months for bevacizumab plus FOLFOX, XELOX, or FOLFIRI and in excess of 9 months for bevacizumab plus a fluoropyrimidine monotherapy.

\section{Bevacizumab in the treatment of metastatic CRC in the second- line setting}

In general, bevacizumab should be integrated with first-line chemotherapy in patients with metastatic CRC. In the case of major contraindications for bevacizumab, such as severe vascular disease or prior arterial thrombotic events, one should make a case for avoiding bevacizumab therapy during all lines of treatment.

In the event a bevacizumab-naïve patient fails first-line chemotherapy, bevacizumab should be strongly considered in the setting of 5-FU based combination therapy in the secondline setting. Supporting evidence comes from ECOG 3200, a phase III study randomizing patients to FOLFOX-4 plus bevacizumab versus FOLFOX-4 in patients who progressed after first-line irinotecan, 5-FU and leucovorin. ${ }^{56}$ The bevacizumab arm was associated with a superior response rate (21.8\% versus 9.2\%), median PFS (7.2 versus 4.8 months), and median OS (12.9 versus 10.8 months). No other randomized phase III studies investigated bevacizumab after failure of a non-bevacizumab-containing regimen. However, it is generally agreed, based on synergy with 5-FU, that bevacizumab should be considered in combination with FOLFIRI in patients who previously failed FOLFOX without bevacizumab.

The continuation of bevacizumab beyond first-line progression on bevacizumab-based regimens is controversial. Retrospective analysis of the large observational BRiTE study registry suggests a role for the continuation of bevacizumab. ${ }^{61}$ In this study, 1953 patients received first-line bevacizumab-based chemotherapy, 1,192 patients progressed and received second-line chemotherapy. The use of secondline bevacizumab as well as the choice of chemotherapy was at the individual investigator's discretion. Patients who continued on bevacizumab along with their cytotoxic secondline treatment experienced a significant improvement in their median OS, whether computed from the start of first- or second-line therapy (31.8 versus 19.9 months, 19.2 versus 9.5 months, respectively). The lack of randomized design and the likely selection bias of good performance status in the bevacizumab continuation arm lead to caution in the interpretation of these results. The ongoing SWOG S0600/iBET study is currently randomizing patients who progressed on FOLFOX plus bevacizumab to irinotecan (with or without 5 -FU)/ cetuximab with or with bevacizumab. This study will hopefully determine the value of bevacizumab continuation after bevacizumab-first line progression.

\section{Bevacizumab in the treatment of metastatic CRC in the third-line setting}

The role of bevacizumab in combination with bolus or infusional 5-FU/LV in the third line was studied in a multicenter phase II trial of 350 patients who had failed both irinotecan- and oxaliplatin based chemotherapy regimens. ${ }^{62}$ A planned efficacy analysis was done on the first 100 enrolled patients. The objective response rate was $4 \%$ based on the investigator's assessment, and $1 \%$ based upon an independent review. Median PFS and OS were 3.5 and 9 months, respectively. Based on this study, the use of third-line 5-FU/LV plus bevacizumab in chemoresistant patients is considered an ineffective treatment.

\section{Combining bevacizumab with other biological agents: dual inhibition of VEGF and EGFR}

Cetuximab and panitumumab are anti-EGFR monoclonal antibodies with proven efficacy in metastatic CRC. Cetuximab, a IgG1 chimeric monoclonal antibody, is approved as a single agent or in combination with irinotecan in irinotecan-resistant metastatic CRC. ${ }^{63}$ Panitumumab, a human IgG1 monoclonal antibody, is approved as monotherapy in patients with metastatic CRC that have failed standard chemotherapy. ${ }^{64}$ Preclinical models have showed a synergistic effect with dual targeting of anti VEGF and EGFR pathways. ${ }^{65-67}$ The combinations of the above monoclonal antibodies with bevacizumab have been studied in metastatic CRC.

The BOND-2 study is a randomized phase II trial comparing cetuximab plus bevacizumab (CB) with and without irinotecan (CBI) in 83 patients with irinotecan- and oxaliplatin-refractory but bevacizumab-naïve metastatic CRC. ${ }^{68}$ The CBI arm had a 37\% response rate, TTP of 7.9 months, and OS of 14.5 months; the biologic-alone arm of CB was also active, with a $20 \%$ response rate, TTP of 5.6 months, and OS of 11.4 months. The toxicity pattern seemed to be similar to that which would be expected from 
the two agents given alone. However these results seem to be in conflict with the subsequent PACCE and CAIRO2 trials in the first-line treatment of metastatic CRC.

The PACCE study investigated the integration of panitumumab in the first-line treatment of metastatic CRC in combination with standard combination chemotherapy. Patients were treated at their physician's choice with a combination of FOLFOX plus bevacizumab or FOLFIRI plus bevacizumab and were randomized to receive panitumumab or nothing in addition to the standard selected therapy. A preplanned efficacy analysis, disappointingly, showed that the dual biological combination arm had a shorter PFS and increased toxicity leading to study discontunuation. ${ }^{69,70}$ Similarly, results from the CAIRO II study, a randomized study of capecitabine/oxaliplatin plus bevacizumab with or without cetuximab was presented at ASCO 2008. Again, the arm including both cetuximab and bevacizumab showed a significant decrease in PFS compared to the control arm. However, besides skin toxicity and a modest increase in diarrhea, no added toxicity was seen with the addition of cetuximab. ${ }^{71}$ Of interest, neither the PACCE nor the CAIRO 2 studies showed an advantage to adding anti-EGFR inhibitors to bevacizumab-based combination in KRAS wild type patients, a population well known to benefit from cetuximab therapy. Furthermore, the detrimental effects from the addition of anti-EGFR therapy were limited to the KRAS mutant population. ${ }^{71-73}$

Currently, the combination of anti-EGFR and anti-VEGF antibody therapy with chemotherapy in metastatic CRC cancer is contraindicated outside a clinical trial setting. CALBG 80405 continues to investigate the combination of FOLFOX or FOLFIRI with cetuximab, or bevacizumab, or cetuximab plus bevacizumab; however, with an amendment to limit accrual to KRAS wild type population.

\section{Role of bevacizumab in the adjuvant setting}

The successful integration of bevacizumab in metastatic CRC paved the way for several adjuvant randomized studies in CRC in the US and Europe. Table 3 lists some of the trials that completed accrual or where accrual is ongoing. ${ }^{74-78}$

The initial safety data on bevacizumab given in conjunction with modified combination fluorouracil and leucovorin and oxaliplatin (mFOLFOX6) as postoperative adjuvant therapy for patients with stage II or stage III colorectal cancer was presented at ASCO 2008. ${ }^{79}$ All wound complications seen in the study were grade $3,1.7 \%$ in the bevacizumab versus
$0.3 \%$ in the control arm. Surgical intervention was required in only one case and in discontinuation of bevacizumab in half of the cases. Efficacy data from randomized studies are still awaited. Until then, the integration of bevacizumab in combination with chemotherapy in the adjuvant setting is not recommended.

\section{Bevacizumab in the elderly (more than 70 years) and in patients with poor performance status}

Treating the elderly CRC population, especially those with associated co-morbidities, is linked with significant challenges. The majority of physicians tend to treat the elderly differently than younger patients, either by reducing dosages or by limiting the number of agents/combinations used in this population. The limited representation of elderly patients, particularly those $\geq 70$ years, in large randomized clinical trials has resulted in a lack of clear identification of an optimal regimen in this subgroup of patients. ${ }^{6,753}$ Furthermore, with the increase in cardiovascular risk as individuals age, concern has naturally risen about the use of bevacizumab in this population.

The BRiTE study is a large $(\mathrm{n}=1953)$ prospective multicenter study of first-line metastatic CRC patients receiving a bevacizumab-based regimen with a follow up of more than 3 years. The objective of the study was to describe the incidence and timing of bevacizumab-related safety outcomes associated with long-time exposure to bevacizumab. ${ }^{80} \mathrm{Of}$ 1,953 patients in the BRiTE study, 896 were $\geq 65$ years ( 533 $65-74,363 \geq 75$ and $161 \geq 80$ years). Apart from a higher rate of arterial thrombotic events (ATE) in older patients, bevacizumab safety in the elderly was comparable to that in patients $<65$ years. Risk factors for developing ATEs included poor performance status and prior history of ATE. PFS in the elderly was similar to that in patients $<65$ years in BRiTE and other pivotal bevacizumab trials. Multivariate analyses revealed that the lower median OS observed in older patients was attributed to poorer performance status and relative underexposure to active cytotoxic agents and post-progression therapy in the elderly.

Bevacizumab has also been investigated in patients with poor performance status (PS 1 or 2) and with advanced age ( $\geq 65$ years), who are not eligible for combination chemotherapy in a prospective randomized phase II study of 5-FU/LV with or without bevacizumab (Table 1). ${ }^{57}$ The bevacizumab arm showed a significant benefit in OS (16.6 versus 12.1 months, $\mathrm{p}=0.160)$, PFS (9.2 versus 5.5 months, $\mathrm{p}=0.0002)$ and RR (26 versus $15.2 \%, \mathrm{p}=0.055)$. 
Table 3 Current adjuvant studies utilizing bevacizumab in colorectal cancer

\begin{tabular}{lll}
\hline Adjuvant studies & Chemotherapy regimen & $\mathbf{n}$ \\
\hline AVANT $^{74}$ & $\begin{array}{l}\text { FOLFOX-4 vs FOLFOX-4 plus } \\
\text { bevacizumab vs XELOX plus } \\
\text { bevacizumab } \\
\text { mFOLFOX6 with and without }\end{array}$ & 3450 \\
NSABP C-08 & 2632 \\
E 52027 & $\begin{array}{l}\text { Folizumab } \\
\text { bevacizumab }\end{array}$ & 3610 \\
QUASAR 2 & $\begin{array}{l}\text { Capecitabine with and without } \\
\text { bevacizumab }\end{array}$ & 2240 \\
\hline
\end{tabular}

In summary, although limited data exist on the use of bevacizumab and chemotherapy in elderly patients with metastatic CRC, the available information suggests that in patients with an adequate performance status, the efficacy of bevacizumab therapy is similar in older patients to that in the general population. In general, it is appropriate to treat patients based on their performance status, rather than just on their numerical age. There are currently no age-related eligibility criteria for treatment with bevacizumab.

\section{Safety profile and management of adverse events associated with bevacizumab}

Early phase II and pivotal phase III studies in CRC utilizing bevacizumab have identified hypertension (11\%-32\%), bleeding (mainly epistaxis; 30\%-53\%), proteinuria (10\%-38\%), ATE (1\%-10\%), gastrointestinal perforation $(0.3 \%-2 \%)$, wound healing $(1.3 \%-3.7 \%)$ as bevacizumabassociated adverse events; these are detailed in Table 4. In most cases these side effects do not overlap with the toxicity associated with cytotoxic chemotherapy. As our clinical experience with bevacizumab accumulates, guidelines for managing the adverse events associated with bevacizumab are increasingly emerging.

\section{Hypertension (HTN) and proteinuria}

Initial phase II trials in metastatic CRC revealed a higher incidence of HTN in patients treated with bevacizumab (grade 3 $16 \%) .{ }^{81}$ Subsequently, the pivotal phase III trial of IFL with or without bevacizumab in metastatic CRC showed similar results (all grade HTN: 22.4\%; grade $3 \mathrm{HTN}$ : 11\%, and grade 4 HTN: $0 \%-1 \%) .{ }^{9}$ The mechanism of bevacizumab-related HTN is unclear and has been attributed to possible alterations in the nitric oxide signaling pathway. ${ }^{82}$ VEGF inhibition by bevacizumab results in vasoconstriction of blood vessels with a resulting increase in blood pressure. Other mechanisms involving the renin-angiotensin system, endothelial dysfunction and capillary rarefaction have also been proposed. ${ }^{83,84}$ There was initial suggestion of a correlation between the dose of bevacizumab and development of HTN and proteinuria. ${ }^{85}$ A phase II study with bevacizumab dosed at $10 \mathrm{mg} / \mathrm{kg}$ was associated with a higher incidence of all grade HTN (28\% versus $11 \%$ ) compared with the standard dose of bevacizumab $(5 \mathrm{mg} / \mathrm{kg}) .{ }^{86}$ However, subsequent phase III studies which used $10 \mathrm{mg} / \mathrm{kg}$ of bevacizumab showed the incidence of HTN to be much lower (grade 3 HTN 5.2\%). ${ }^{56}$

There are no clear guidelines for management of hypertension in patients receiving bevacizumab. Hypertension can occur at any time after initiation of therapy with bevacizumab; hence blood pressure should be checked before each cycle of treatment. Patients should also be encouraged to monitor blood pressure at home. Patients who develop grade 2 HTN during bevacizumab therapy should be managed using standard oral antihypertensive therapy. Grade 3 HTN is managed by titrating up the patients' anti-HTN medication or may require combination therapy with standard antihypertensive medications (typically angiotensin-converting enzyme [ACE] inhibitors, diuretics, calcium channel or beta blockers). Although ACE inhibitors are attractive, especially with concomitant renal dysfunction or proteinuria, currently there is no data to determine the best antihypertensive agent available. ${ }^{87-89}$ In case of uncontrolled hypertension not responding to oral medications, bevacizumab should be suspended or permanently discontinued. ${ }^{87,88}$ Grade 4 hypertension is an infrequent occurrence, and in patients who develop hypertensive crisis (grade 4) bevacizumab is permanently discontinued..$^{87,88}$

Incidence of grade $3 / 4$ proteinuria in recent phase III trials with bevacizumab in metastatic CRC have been reported in the range of less than $2 \% .{ }^{9,55,90}$ The mechanism of proteinuria is not fully understood, but may be related to the effects of VEGF on the renal glomerular capillaries. It is advisable that patients receiving bevacizumab get a urinalysis (regular dipstick or microscopic) at baseline and throughout the treatment. Patients with proteinuria $\geq 2+$ on dipstick should have a 24-hour urine check for quantification of protein. If patients develop $\geq 2 \mathrm{~g} / 24$ hours proteinuria, bevacizumab administration should be interrupted until proteinuria improves to $<2 \mathrm{~g} / 24$ hours .Patients should be evaluated for ACE inhibitors or angiotensin-receptor blocker as initial treatment in case of development of proteinuria with hypertension. Bevacizumab should be permanently discontinued in patients 


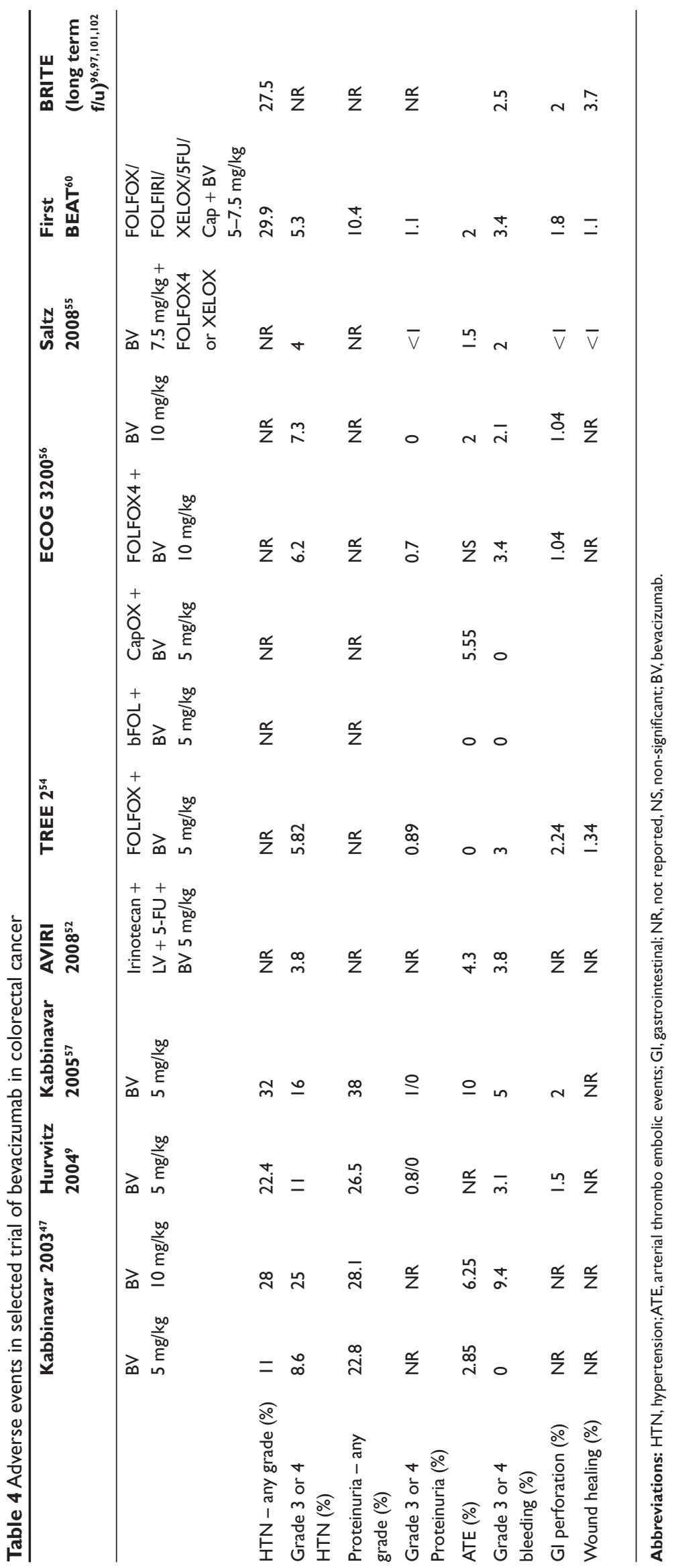


developing nephrotic syndrome. ${ }^{87-89}$ Nephrotic syndrome occurred in seven of $1459(0.5 \%)$ patients receiving bevacizumab in clinical studies. One patient died and one required dialysis. In three patients, proteinuria decreased in severity several months after discontinuation of bevacizumab.

\section{Thromboembolism}

Patients with malignancies are more prone to develop thromboembolic complications when compared to the normal population. Early phase II trials of bevacizumab in metastatic CRC showed a higher incidence of arterial thromboembolism (ATE) (5\%) but not venous thromboembolism (VTE) (10\%) compared with the control arm (3\% and 9\% respectively) ${ }^{57}$ In the pivotal phase III trial in patients with metastatic CRC evaluating the IFL regimen with and without bevacizumab, the incidence of all venous and ATE was $19.4 \%$ versus $16.2 \%$ in the non-bevacizumab arm. ${ }^{9}$ It has been speculated that the blockade of the VEGF receptors leads to apoptosis of endothelial cells in the vasculature, thus exposing the subendothelial collagen and initiating a coagulation cascade resulting in an increased risk for thrombus formation. ${ }^{91}$

Analysis of pooled data from five randomized trials in patients with metastatic CRC, breast, and non-small-cell lung cancer by Scappaticci et al showed increased risk of ATE (HR 2.0, 95\% CI 1.05-3.75, p = 0.031) but not VTE (HR 0.89, 95\% CI 0.66-1.20, $\mathrm{p}=0.44) .{ }^{92}$ The absolute rate of developing an ATE was 5.5 events per 100 person-years for patients receiving combination therapy versus 3.1 events for patients receiving chemotherapy alone (ratio 1.8, 95\% CI $0.94-3.33, p=0.76$ ). It was also noted that patients who do not use aspirin had a higher risk of developing ATE compared to the group taking aspirin (3.6\% versus $1.2 \%)$. In the subgroup analysis, patients older then 65 years with previous history of ATE had a significantly higher risk of developing ATE, if not taking concurrent aspirin with bevacizumab (22.9\% versus $3.4 \%$ ). The difference between older and younger than 65 years was not significant in those receiving aspirin (12.5\% versus 0\%). In August 2004 the US Food and Drug Administration (FDA) sent a Dear Doctor letter to all US oncologists describing the increased rate of ATE in patients with prior events of ATE or patients aged $>65$ years during bevacizumab therapy.

Hambleton et al assessed the outcomes of patients with metastatic CRC who had a thrombotic event while receiving treatment with bevacizumab and remained on study with anticoagulation. ${ }^{93}$ Use of concomitant full-dose anticoagulation therapy with bevacizumab in combination with chemotherapy did not increase the risk of hemorrhagic complications in patients with metastatic CRC.

Analysis of thrombotic events in the BRiTE registry showed a similar risk of developing ATE in patients $<65$ years old compared with patients in the age group of 65 to 74 years $(1.5 \%)$, though the rate was higher in patients older then 75 years of age $(4.1 \%) .{ }^{94}$

In summary, patients treated with bevacizumab are at a higher risk of developing ATE, especially patients older then 65 years with previous history of ATE. ${ }^{81,94,95}$ Although patients above 65 years have a slightly higher risk of ATE, oncologists must assess the risk/benefit of its use, especially as the benefit of bevacizumab is seen across all age groups. Use of prophylactic low-dose aspirin and treatment of VTE with full anticoagulation with warfarin appear safe. ${ }^{81,93}$ In patients who develop VTE on bevacizumab, bevacizumab should be on hold until the patient is fully anticoagulated; and in the event of any life-threatening VTE, bevacizumab should be discontinued. Patients who had a recent ATE (myocardial infarction, cerebrovascular event, transient ischemic attack) bevacizumab should not be initiated for at least 6 months. Bevacizumab should be started only once patient is stable, asymptomatic and after a detailed discussion of the risk involved. Patients who develop any ATE during bevacizumab therapy should be taken off treatment. ${ }^{87-89}$ Patients who develop venous thromboembolism can be safely treated with anticoagulant therapy and there does not appear to be increased risk of bleeding with concomitant use of bevacizuamb.

As physicians gain more experience with bevacizumab and become familiar with patient selection, the risk of ATE associated with bevacizumab appears to decline. Appropriately selected patients on E3200 and NO16966 studies with FOLFOX and bevacizumab experienced an ATE rate of $0.9 \%$ and $2 \%$, respectively. These did not differ significantly from the rates of their chemotherapy-only comparative arms of $0.4 \%$ and $1 \%$, respectively. ${ }^{55,56}$

\section{Bleeding}

Increased of bleeding was seen in patients with metastatic CRC treated with bevacizumab in early phase II studies. ${ }^{81}$ The most significant were gastrointestinal bleeding $(6 \%)$ and the most common was self-limited epistaxis (46\%). Bleeding from mucocutaneous membranes is common with bevacizumab and occurs in $20 \%$ to $40 \%$ of patients. This is mainly epistaxis and responds to usual first aid techniques with cessation of most bleeding in less than 5 minutes. Bevacizumab should be discontinued in patients who need 
medical intervention. In randomized phase III studies grade $3-4$ bleeding was seen in $1.8 \%$ to $3.8 \%$ of patients (Table 5 ). This seems to be in line with the data presented from the BRiTE registry which reported serious bleeding events in $2.5 \%(49 / 1953)$ patients treated with bevacizumab and chemotherapy in metastatic CRC patients. ${ }^{96,97}$ The BRiTE study did not exclude patients on antiplatelet therapy(11.2\%) and full dose anticoagulation (6.8\%) and the authors concluded that inclusion of these patients is relatively safe in terms of risk of serious bleeding complications. Patients with central nervous system metastasis are usually excluded from clinical trials, so incidence of CNS hemorrhage in patients with CNS metastasis is unclear.

Bevacizumab should not be initiated in patients with recent hemoptysis or CNS metastasis. Initiation of full dose anticoagulation appears to be safe in terms of risk of serious bleeding events. ${ }^{81,93,96}$ Patients with congenital or acquired bleeding diathesis should be started on bevacizumab with caution. ${ }^{87,88}$

\section{Surgical wound healing complications}

Angiogenesis is an essential component of normal wound healing and VEGF inhibitors can delay angiogenesis in patients with cancer. ${ }^{98}$ Wound healing complications were analyzed in patients who underwent surgery within 28 to 60 days of starting bevacizumab treatment and while on bevacizumab treatment in the study by Hurwitz et al. ${ }^{99}$ Patients who underwent surgery within 28 to 60 days of receiving bevacizumab with IFL or with 5 -FU, had minimal increase in grade 3-4 wound healing/bleeding compared with the IFL-only group $\left(10 \%, 6.8 \%\right.$ and $0 \%$ respectively). ${ }^{99}$ Scappaticci et al assessed wound healing complications in patients with metastatic CRC who underwent cancer surgery 28 to 60 days before treatment and in patients who underwent major surgery during treatment with 5-FU/LV with and without bevacizumab. ${ }^{100}$ Cases were reviewed for wound healing complications occurring $\leq 60$ days after surgery.
Increased wound healing complications were seen in patients who underwent surgery during bevacizumab therapy $(13 \%$ versus $3.4 \%$ ), but not in patients who received bevacizumab 28 to 60 days post operatively $(1.3 \%$ versus $0.5 \%)$. The wound complications included anastomotic wound dehiscence, ecchymosis and bleeding. Data from the BRiTE study showed an overall rate of serious wound healing complication as $3.7 \%(23 / 622)$, which was lower than the initial report in the pivotal by Hurwitz et al. ${ }^{101}$ Patients requiring surgery within 14 days of last bevacizumab dose had an increased risk of postoperative complications compared with patients who had surgery after 60 days $(6.5 \%$ versus $1.8 \%) .{ }^{102}$ It is of note, however, that the implantation of a venous access device shortly before starting bevacizumab treatment does not seem to result in increased bleeding or other complications. ${ }^{103}$

In summary, patients who underwent surgery during or within 14 days of bevacizumab are at a higher risk of developing wound healing complications. Based on available data, bevacizumab should not be initiated within 30 days after surgery. Minor surgical procedures, such as insertion of venous access devices, can be done within 7 days of bevacizumab treatment. If patients have to undergo emergency surgery, bevacizumab should be held and patients should be followed closely for complications. Elective surgeries should be planned 6 to 8 weeks after last dose of bevacizumab, though chemotherapy alone can be continued 2 to 3 weeks before surgery. Bevacizumab should be discontinued if any wound complication (fistula, dehiscence) develops or if an intra-abdominal abscess develops.

\section{Gastrointestinal (GI) perforation}

The incidence of GI perforation is seen in $1.5 \%$ to $2.4 \%$ of metastatic CRC patients treated with bevacizumab and is associated with the highest bevacizumab-associated mortality (Table 5). The incidence of GI perforations appears to be higher in patients with intact primary tumor, prior adjuvant radiation therapy in rectal cancer, long-term nonsteroidal

Table 5 Common terminology criteria for adverse events v3.0 (CTCAE) ${ }^{109}$

\begin{tabular}{|c|c|c|c|c|c|}
\hline & Grade I & Grade 2 & Grade 3 & Grade 4 & Grade 5 \\
\hline Hypertension & $\begin{array}{l}\text { Asymptomatic, transient } \\
(<24 \mathrm{~h}) \text { increase by } \\
>20 \mathrm{mmHg} \text { (diastolic) or } \\
\text { to }>150 / 100 \text { if previously } \\
\text { within normal limits }\end{array}$ & $\begin{array}{l}\text { Recurrent or persistent } \\
(>24 \mathrm{~h} \text { ) or symptomatic } \\
\text { increase by }>20 \mathrm{mmHg} \\
\text { (diastolic) or to }>150 / 100 \\
\text { if previously within normal } \\
\text { limits }\end{array}$ & $\begin{array}{l}\text { Requiring more then } \\
\text { one drug or more } \\
\text { intensive therapy than } \\
\text { previously }\end{array}$ & $\begin{array}{l}\text { Life-threatening } \\
\text { consequences } \\
\text { (hypertensive crisis) }\end{array}$ & Death \\
\hline Proteinuria & $\mathrm{I}+$ or $0.15-1.0 \mathrm{~g} / 24 \mathrm{~h}$ & $2+$ to $3+$ or $>1.0-3.5 \mathrm{~g} / 24 \mathrm{~h}$ & $4+$ or $>3.5 \mathrm{~g} / 24 \mathrm{~h}$ & Nephrotic syndrome & Death \\
\hline Thrombo-embolism & - & DVT or cardiac thrombosis & DVT or thrombosis & $\begin{array}{l}\text { Embolic event including } \\
\text { PE or life-threatening } \\
\text { thrombus }\end{array}$ & Death \\
\hline
\end{tabular}


anti-inflammatory drug (NSAID) therapy ( $\geq 1$ month of use), peptic ulcer disease, diverticulosis, and previous gastrointestinal surgery. ${ }^{9,104,105}$ Recently presented BRiTE registry data showed an incidence of GI perforation of $2.5 \%$ in patients $<65$ years, $1.5 \%$ in patients 65 to 74 years, $1.1 \%$ in patients $\geq 75$ years old and $0.6 \%$ in patients $\geq 80$ years old. ${ }^{94}$ In spite of surgery, treatment mortality rate remains high $(12.5 \%-30 \%)$ for patients who develop GI perforation while receiving bevacizumab treatment. ${ }^{87,106}$

Patients with metastatic CRC who are treated with bevacizumab should be closely monitored for signs and symptoms of GI perforation, and radiographic imaging should be obtained immediately when clinically suspected. Patients with active peptic ulcer disease should be treated with either $\mathrm{H} 2$ blocker or proton pump inhibitors. Bevacizumab should be permanently discontinued in patients who develop GI perforation. Surgical intervention should be considered as and when appropriate. ${ }^{87,88}$ The manufacturer has issued a black box warning about the risk of GI perforation, wound dehiscence and fatal hemoptysis.

\section{Reversible posterior leukoencephalopathy syndrome (RPLS)}

RPLS has been reported in clinical studies (with an incidence of $<0.1 \%$ ) and in postmarketing experience. ${ }^{87}$ RPLS is a neurological disorder which can present with headache, seizure, lethargy, confusion, blindness and other visual and neurologic disturbances. Mild to severe hypertension may be present, but is not necessary for diagnosis of RPLS. Magnetic resonance imaging is necessary to confirm the diagnosis of RPLS. ${ }^{107,108}$ The onset of symptoms has been reported to occur from 16 hours to 1 year after initiation of bevacizumab. Discontinuation of bevacizumab and aggressive management of hypertension is indicated in patients developing RPLS. Symptoms usually resolve or improve within days, although some patients have experienced ongoing neurologic sequelae. The safety of reinitiating bevacizumab therapy in patients previously experiencing RPLS is not known. ${ }^{87}$

\section{Conclusion}

The integration of bevacizumab in the management of CRC has resulted in major improvement benefits in patients with metastatic disease. In general, bevacizumab should be integrated with first-line chemotherapy in patients with metastatic CRC, unless contraindicated. The continuation of bevacizumab beyond first-line progression is still controversial, due to lack of prospective randomized evidence in this setting. The combination of anti-EGFR antibodies and bevacizumab with chemotherapy in metastatic CRC is currently contraindicated outside a clinical trial setting. Older patients do benefit from the addition of bevacizumab to chemotherapy, albeit with a slightly increased incidence of ATE.

Early results from adjuvant bevacizumab-based studies therapy of colon cancer do not show any significantly increased risks. However, until supportive clinical efficacy data become available in that setting, the use of bevacizumab should be considered investigational.

The recommended dosing of bevacizumab is $5 \mathrm{mg} / \mathrm{kg}$ every 2 weeks with FOLFOX/FOLFIRI-based regimens and $7.5 \mathrm{mg} / \mathrm{kg}$ every 3 weeks with XELOX in the first-line setting. Bevacizumab is generally well tolerated in combination with chemotherapy, without additive increase in chemotherapyrelated toxicities. However, bevacizumab-related adverse events such as HTN, proteinuria, perforation and ATE require extensive patient counseling and monitoring.

\section{Disclosures}

The authors have no conflicts of interest to disclose.

\section{References}

1. Jemal A Siegel R, Ward E. Cancer statistics, 2008. CA Cancer J Clin. 2008;58(2):71-96.

2. Ries LAG, Melbert D, et al. SEER Cancer Statistics Review, 1975-2005, National Cancer Institute. Bethesda, MD, http://seer.cancer. gov/csr/1975_2005/, based on November 2007 SEER data submission, posted to the SEER web site, 2008.

3. Cancer.net. Colorectal Cancer - Overview.Available at http://www. cancer.net/patient/Cancer+Types/Colorectal+Cancer. In.

4. Sanoff HK, Sargent DJ, Campbell ME, et al. N9741: Survival update and prognostic factor analysis of oxaliplatin (Ox) and irinotecan (Iri) combinations for metastatic colorectal cancer (MCRC). J Clin Oncol. (Meeting Abstracts) 2007;25(18 suppl):4067.

5. de Gramont A, Figer A, Seymour M, et al. Leucovorin and fluorouracil with or without oxaliplatin as first-line treatment in advanced colorectal cancer. J Clin Oncol. 2000;18(16):2938-47.

6. Douillard JY, Cunningham D, Roth AD, et al. Irinotecan combined with fluorouracil compared with fluorouracil alone as first-line treatment for metastatic colorectal cancer: a multicentre randomised trial. Lancet. 2000;355(9209):1041-7.

7. Saltz LB, Cox JV, Blanke C, et al. Irinotecan plus fluorouracil and leucovorin for metastatic colorectal cancer. Irinotecan Study Group. N Engl J Med. 2000;343(13):905-14.

8. Fuchs CS, Marshall J, Mitchell E, et al. Randomized, controlled trial of irinotecan plus infusional, bolus, or oral fluoropyrimidines in firstline treatment of metastatic colorectal cancer: results from the BICC-C Study. J Clin Oncol. 2007;25(30):4779-86.

9. Hurwitz H, Fehrenbacher L, Novotny W, et al. Bevacizumab plus irinotecan, fluorouracil, and leucovorin for metastatic colorectal cancer. N Engl J Med. 2004;350(23):2335-42.

10. Cunningham D, Humblet Y, Siena S, et al. Cetuximab monotherapy and cetuximab plus irinotecan in irinotecan-refractory metastatic colorectal cancer. N Engl J Med. 2004;351(4):337-45.

11. Grothey A, Sargent D. Overall survival of patients with advanced colorectal cancer correlates with availability of fluorouracil, irinotecan, and oxaliplatin regardless of whether doublet or single-agent therapy is used first Line. J Clin Oncol. 2005;23(36):9441-2. 
12. Fidler IJ Ellis LM. The implications of angiogenesis for the biology and therapy of cancer metastasis. Cell. 1994;79:185-8.

13. Folkman J. Tumor angiogenesis: therapeutic implications. N Engl J Med. 1971;285(21):1182-6.

14. Ferrara N, Gerber HP, LeCouter J. The biology of VEGF and its receptors. Nat Med. 2003;9(6):669-76.

15. Ferrara N. Role of vascular endothelial growth factor in regulation of physiological angiogenesis. Am J Physiol. 2001;280(6):C1358-66.

16. Dvorak HF. Discovery of vascular permeability factor (VPF). Exp Cell Res. 2006;312(5):522-6.

17. Nagy JA, Vasile E, Feng D, et al. VEGF-A induces angiogenesis, arteriogenesis, lymphangiogenesis, and vascular malformations. Cold Spring Harb Symp Quant Biol. 2002;67:227-37.

18. Nagy JA, Dvorak AM, Dvorak HF. VEGF-A(164/165) and PIGF: roles in angiogenesis and arteriogenesis. Trends Cardiovasc Med. 2003;13(5):169-75.

19. Dvorak HF. Vascular permeability factor/vascular endothelial growth factor: a critical cytokine in tumor angiogenesis and a potential target for diagnosis and therapy. J Clin Oncol. 2002;20(21):4368-80.

20. Tanaka K, Yamaguchi S, Sawano A, Shibuya M. Characterization of the extracellular domain in vascular endothelial growth factor receptor-1 (Flt-1 tyrosine kinase). Jpn J Cancer Res. 1997;88(9):867-76.

21. Shinkai A, Ito M, Anazawa H, Yamaguchi S, Shitara K, Shibuya M. Mapping of the sites involved in ligand association and dissociation at the extracellular domain of the kinase insert domain-containing receptor for vascular endothelial growth factor. $J$ Biol Chem. 1998;273(47):31283-8.

22. Shibuya M. Vascular endothelial growth factor receptor-2: its unique signaling and specific ligand, VEGF-E. Cancer Sci. 2003;94(9):751-6.

23. Shibuya M. Structure and function of VEGF/VEGF-receptor system involved in angiogenesis. Cell Struct Func. 2001;26(1):25-35.

24. Shibuya M. [Involvement of the VEGF-Flt-receptor family in angiogenesis]. Nippon Yakurigaku Zasshi. 1996;107(3):119-31.

25. Ogawa S, Oku A, Sawano A, Yamaguchi S, Yazaki Y, Shibuya M. A novel type of vascular endothelial growth factor, VEGF-E (NZ-7 VEGF), preferentially utilizes KDR/Flk-1 receptor and carries a potent mitotic activity without heparin-binding domain. $J$ Biol Chem. 1998;273(47):31273-82.

26. Wang L, Zeng H, Wang P, Soker S, Mukhopadhyay D. Neuropilin1-mediated vascular permeability factor/vascular endothelial growth factor-dependent endothelial cell migration. $J$ Biol Chem. 2003;278(49):48848-60.

27. Soker S, Takashima S, Miao HQ, Neufeld G, Klagsbrun M. Neuropilin-1 is expressed by endothelial and tumor cells as an isoform-specific receptor for vascular endothelial growth factor. Cell. 1998;92(6):735-45.

28. Soker S. Neuropilin in the midst of cell migration and retraction. Int $J$ Biochem Cell Biol. 2001;33(4):433-7.

29. Halder JB, Zhao X, Soker S, et al. Differential expression of VEGF isoforms and VEGF(164)-specific receptor neuropilin-1 in the mouse uterus suggests a role for $\operatorname{VEGF}(164)$ in vascular permeability and angiogenesis during implantation. Genesis. 2000;26(3):213-24.

30. Zafirellis K, Agrogiannis G, Zachaki A, Gravani K, Karameris A, Kombouras C. Prognostic significance of VEGF expression evaluated by quantitative immunohistochemical analysis in colorectal cancer. J Surg Res. 2008;147(1):99-107.

31. Takebayashi Y, Aklyama S, Yamada K, Akiba S, Aikou T. Angiogenesis as an unfavorable prognostic factor in human colorectal carcinoma Cancer. 1996;78(2):226-31.

32. Takebayashi Y, Akiyama S, Akiba S, et al. Clinicopathologic and prognostic significance of an angiogenic factor, thymidine phosphorylase, in human colorectal carcinoma. J Natl Cancer Inst. 1996;88(16):1110-7.

33. Logan-Collins JM, Lowy AM, Robinson-Smith TM, et al. VEGF expression predicts survival in patients with peritoneal surface metastases from mucinous adenocarcinoma of the appendix and colon. Ann Surg Oncol. 2008;15(3):738-44.
34. Lee JC, Chow NH, Wang ST, Huang SM. Prognostic value of vascular endothelial growth factor expression in colorectal cancer patients. Eur $J$ Cancer. 2000;36(6):748-53.

35. Melnyk O, Shuman MA, Kim KJ. Vascular endothelial growth factor promotes tumor dissemination by a mechanism distinct from its effect on primary tumor growth. Cancer Res. 1996;56(4):921-4.

36. Kim KJ, Li B, Winer J, et al. Inhibition of vascular endothelial growth factor-induced angiogenesis suppresses tumour growth in vivo. Nature. 1993;362(6423):841-4.

37. Warren RS, Yuan H, Matli MR, Gillett NA, Ferrara N. Regulation by vascular endothelial growth factor of human colon cancer tumorigenesis in a mouse model of experimental liver metastasis. $J$ Clin Invest. 1995;95(4):1789-97.

38. Klement G, Baruchel S, Rak J, et al. Continuous low-dose therapy with vinblastine and VEGF receptor-2 antibody induces sustained tumor regression without overt toxicity. J Clin Invest. 2000;105(8):R15-24.

39. Lee CG, Heijn M, di Tomaso E, et al. Anti-Vascular endothelial growth factor treatment augments tumor radiation response under normoxic or hypoxic conditions. Cancer Res. 2000;60(19):5565-70.

40. Kozin SV, Boucher Y, Hicklin DJ, Bohlen P, Jain RK, Suit HD. Vascular endothelial growth factor receptor-2-blocking antibody potentiates radiation-induced long-term control of human tumor xenografts. Cancer Res. 2001;61(1):39-44.

41. Margolin K, Gordon MS, Holmgren E, et al. Phase Ib trial of intravenous recombinant humanized monoclonal antibody to vascular endothelial growth factor in combination with chemotherapy in patients with advanced cancer: pharmacologic and long-term safety data. $J$ Clin Oncol. 2001;19(3):851-6.

42. Gordon MS, Margolin K, Talpaz M, et al. Phase I safety and pharmacokinetic study of recombinant human anti-vascular endothelial growth factor in patients with advanced cancer. J Clin Oncol. 2001;19(3):843-50.

43. Cobleigh MA, Langmuir VK, Sledge GW, et al. A phase I/II doseescalation trial of bevacizumab in previously treated metastatic breast cancer. Semin Oncol. 2003;30(5 Suppl 16):117-24.

44. Yang JC, Haworth L, Sherry RM, et al. A randomized trial of bevacizumab, an anti-vascular endothelial growth factor antibody, for metastatic renal cancer. $N$ Engl J Med. 2003;349(5):427-34.

45. Kindler HL, Friberg G, Singh DA, et al. Phase II trial of bevacizumab plus gemcitabine in patients with advanced pancreatic cancer. J Clin Oncol. 2005;23(31):8033-40.

46. Varker KA, Biber JE, Kefauver C, et al. A randomized phase 2 trial of bevacizumab with or without daily low-dose interferon alfa- $2 \mathrm{~b}$ in metastatic malignant melanoma. Ann Surg Oncol. 2007;14(8):2367-76.

47. Kabbinavar F, Hurwitz HI, Fehrenbacher L, et al. Phase II, randomized trial comparing bevacizumab plus fluorouracil (FU)/leucovorin (LV) with $\mathrm{FU} / \mathrm{LV}$ alone in patients with metastatic colorectal cancer. $J$ Clin Oncol. 2003;21(1):60-5.

48. Kopetz S, Glover KY, Eng C, et al. Phase II study of infusional 5 -fluorouracil, leucovorin, and irinotecan (FOLFIRI) plus bevacizumab as first-line treatment for metastatic colorectal cancer. J Clin Oncol. (Meeting Abstracts) 2007;25(18 suppl):4089.

49. Sobrero A, Ackland S, Carrion RP, et al. Efficacy and safety of bevacizumab in combination with irinotecan and infusional 5-FU as first-line treatment for patients with metastatic colorectal cancer. J Clin Oncol. (Meeting Abstracts) 2006;24(18 suppl):3544.

50. Fuchs CS, Marshall J, Barrueco J. Randomized, controlled trial of irinotecan plus infusional, bolus, or oral fluoropyrimidines in first-line treatment of metastatic colorectal cancer: updated results from the BICC-C study. J Clin Oncol. 2008;26(4):689-90.

51. Fuchs CS, Marshall J, Mitchell E, et al. Randomized, controlled trial of irinotecan plus infusional, bolus, or oral fluoropyrimidines in firstline treatment of metastatic colorectal cancer: results from the BICC-C Study. J Clin Oncol. 2007;25(30):4779-86.

52. Ackland SP, Clarke S, Perez-Carrión R, et al. Updated efficacy data from AVIRI: A large phase IV trial of first-line bevacizumab plus FOLFIRI in patients with mCRC. Gastrointestinal Cancers Symposium 2008; Orlando; 2008. Abstract No:463. 
53. Goldberg RM, Sargent DJ, Morton RF, et al. A randomized controlled trial of fluorouracil plus leucovorin, irinotecan, and oxaliplatin combinations in patients with previously untreated metastatic colorectal cancer. J Clin Oncol. 2004;22(1):23-30.

54. Hochster HS, Hart LL, Ramanathan RK, et al. Safety and efficacy of oxaliplatin and fluoropyrimidine regimens with or without bevacizumab as first-line treatment of metastatic colorectal cancer: Results of the TREE Study. J Clin Oncol. 2008;26(21):3523-9.

55. Saltz LB, Clarke S, Diaz-Rubio E, et al. Bevacizumab in combination with oxaliplatin-based chemotherapy as first-line therapy in metastatic colorectal cancer: a randomized phase III study. J Clin Oncol. 2008;26(12):2013-19.

56. Giantonio BJ, Catalano PJ, Meropol NJ, et al. Bevacizumab in combination with oxaliplatin, fluorouracil, and leucovorin (FOLFOX4) for previously treated metastatic colorectal cancer: results from the Eastern Cooperative Oncology Group Study E3200. J Clin Oncol. 2007;25(12):1539-44.

57. Kabbinavar FF, Schulz J, McCleod M, et al. Addition of bevacizumab to bolus fluorouracil and leucovorin in first-line metastatic colorectal cancer: results of a randomized phase II trial. J Clin Oncol. 2005;23(16):3697-705.

58. Tournigand C, Cervantes A, Figer A, et al. OPTIMOX1: A randomized study of FOLFOX4 or FOLFOX7 with oxaliplatin in a stop-and-go fashion in advanced colorectal cancer - a GERCOR Study. J Clin Oncol. 2006;24(3):394-400.

59. Grothey A, Hart LL, Rowland KM, et al. Intermittent oxaliplatin (oxali) administration and time-to-treatment-failure (TTF) in metastatic colorectal cancer (mCRC): Final results of the phase III CONcePT trial. J Clin Oncol. (Meeting Abstracts) 2008;26(15 suppl):4010.

60. Berry SR, Van Cutsem E, Kretzschmar A, et al. Final efficacy results for bevacizumab plus standard first-line chemotherapies in patients with metastatic colorectal cancer: First BEAT. J Clin Oncol. (Meeting Abstracts) 2008;26(15 suppl):4025.

61. Grothey A, Sugrue M, Hedrick E, et al. Association between exposure to bevacizumab (BV) beyond first progression (BBP) and overall survival (OS) in patients (pts) with metastatic colorectal cancer (mCRC): Results from a large observational study (BRiTE). J Clin Oncol. (Meeting Abstracts) 2007;25(18 suppl):4036.

62. Chen HX, Mooney M, Boron M, et al. Phase II multicenter trial of bevacizumab plus fluorouracil and leucovorin in patients with advanced refractory colorectal cancer: an NCI Treatment Referral Center Trial TRC-0301. J Clin Oncol. 2006;24(21):3354-60.

63. Cunningham D, Humblet $Y$, Siena S, et al. Cetuximab monotherapy and cetuximab plus irinotecan in irinotecan-refractory metastatic colorectal cancer. $N$ Engl J Med. 2004;351(4):337-45.

64. Van Cutsem E, Peeters M, Siena S, et al. Open-label phase III trial of panitumumab plus best supportive care compared with best supportive care alone in patients with chemotherapy-refractory metastatic colorectal cancer. J Clin Oncol. 2007;25(13):1658-64.

65. Ciardiello F, Bianco R, Damiano V, et al. Antiangiogenic and antitumor activity of anti-epidermal growth factor receptor $\mathrm{C} 225$ monoclonal antibody in combination with vascular endothelial growth factor antisense oligonucleotide in human GEO colon cancer cells. Clin Cancer Res. 2000;6(9):3739-47.

66. Jung YD, Mansfield PF, Akagi M, et al. Effects of combination antivascular endothelial growth factor receptor and anti-epidermal growth factor receptor therapies on the growth of gastric cancer in a nude mouse model. Eur J Cancer. 2002;38(8):1133-40.

67. Shaheen RM, Ahmad SA, Liu W, et al. Inhibited growth of colon cancer carcinomatosis by antibodies to vascular endothelial and epidermal growth factor receptors. $\mathrm{Br} J$ Cancer. 2001;85(4):584-9.

68. Saltz LB, Lenz HJ, Kindler HL, et al. Randomized phase II trial of cetuximab, bevacizumab, and irinotecan compared with cetuximab and bevacizumab alone in irinotecan-refractory colorectal cancer: the BOND-2 study. J Clin Oncol. 2007;25(29):4557-61.
69. Hecht JR, Mitchell E, Chidiac T, et al. An updated analysis of safety and efficacy of oxaliplatin (Ox)/bevacizumab (bev) $+/$ - panitumumab (pmab) for first-line treatment (tx) of metastatic colorectal cancer (mCRC) from a randomized, controlled trial (PACCE). 2008 Gastrointestinal Cancers Symposium Orlando. Abstract No: 273.

70. Hecht JR, Mitchell E, Chidiac T, et al. Interim results from PACCE: Irinotecan (Iri)/bevacizumab (bev) \pm panitumumab (pmab) as first-line treatment (tx) for metastatic colorectal cancer (mCRC). 2008 Gastrointestinal Cancers Symposium. Abstract No:279.

71. Punt CJ, Tol J, Rodenburg CJ, et al. Randomized phase III study of capecitabine, oxaliplatin, and bevacizumab with or without cetuximab in advanced colorectal cancer (ACC), the CAIRO2 study of the Dutch Colorectal Cancer Group (DCCG). J Clin Oncol. (Meeting Abstracts) 2008;26(15 suppl):LBA4011.

72. Bokemeyer C, Bondarenko I, Hartmann JT, et al. KRAS status and efficacy of first-line treatment of patients with metastatic colorectal cancer (mCRC) with FOLFOX with or without cetuximab: The OPUS experience. J Clin Oncol. (Meeting Abstracts) 2008;26(15 suppl):4000.

73. Van Cutsem E, Lang I, D'Haens G, et al. KRAS status and efficacy in the first-line treatment of patients with metastatic colorectal cancer (mCRC) treated with FOLFIRI with or without cetuximab: The CRYSTAL experience. J Clin Oncol. (Meeting Abstracts) 2008;26(15 suppl):2.

74. A randomized, three arm multinational phase III study to investigate bevacizumab ( $\mathrm{q} 3 \mathrm{w}$ or $\mathrm{q} 2 \mathrm{w}$ ) in combination with either intermittent capecitabine plus oxaliplatin (XELOX) (q3w) or fluorouracil/leucovorin with oxaliplatin (FOLFOX-4) versus FOLFOX-4 regimen alone as adjuvant chemotherapy in colon carcinoma. Accessed on August 21 at http://www.clinicaltrials.gov/ct2/show/NCT00112918?term=AVANT $\&$ rank $=1$.

75. A phase III clinical trial comparing infusional 5-fluorouracil (5-FU), leucovorin, and oxaliplatin (mFOLFOX6) every two weeks with bevacizumab to the same regimen without bevacizumab for the treatment of patients with resected stages II and III carcinoma of the colon. Accessed Aug 2008 at http://www.clinicaltrials.gov/ct2/show/ NCT00096278?term=NSABP+C-08\&rank=1.

76. A randomized phase III study comparing 5-FU, leucovorin and oxaliplatin versus $5-\mathrm{FU}$, leucovorin, oxaliplatin and bevacizumab in patients with stage II colon cancer at high risk for recurrence to determine prospectively the prognostic value of molecular markers. 2008. Accessed August 1st, 2008, at http://www.clinicaltrials.gov/ct2/show/ NCT00217737?term=E5202\&rank=1.

77. Giantonio BJ CP, Meropol NJ, et al. Bevacizumab in combination with oxaliplatin, fluorouracil, and leucovorin (FOLFOX4) for previously treated metastatic colorectal cancer: results from the Eastern Cooperative Oncology Group Study E3200. J Clin Oncol. 25:1539-44.

78. A multicentre international study of capecitabine \pm bevacizumab as adjuvant treatment of colorectal cancer. 2008. (Accessed August 1st, 2008, at http://www.octo-oxford.org.uk/alltrials/trials/q2.html.

79. Allegra CJ, Yothers G, O'Connell MJ, Sharif S, Wolmark N. Initial safety report of NSABP C-08, a randomized phase III study of modified 5-fluorouracil (5-FU)/leucovorin (LCV) and oxaliplatin (OX) (mFOLFOX6) with or without bevacizumab (bev) in the adjuvant treatment of patients with stage II/III colon cancer. J Clin Oncol. (Meeting Abstracts) 2008;26(15 suppl):4006.

80. Kabbinavar FF, Hambleton J, Mass RD, Hurwitz HI, Bergsland E, Sarkar S. Combined analysis of efficacy: the addition of bevacizumab to fluorouracil/leucovorin improves survival for patients with metastatic colorectal cancer. J Clin Oncol. 2005;23(16):3706-12.

81. Kabbinavar FF, Hambleton J, Mass RD, Hurwitz HI, Bergsland E, Sarkar S. Combined analysis of efficacy: the addition of bevacizumab to fluorouracil/leucovorin improves survival for patients with metastatic colorectal cancer. J Clin Oncol. 2005;23(16):3706-12.

82. Shen B-Q, Lee DY, Zioncheck TF. Vascular endothelial growth factor governs endothelial nitric-oxide synthase expression via a KDR/Flk-1 receptor and a protein kinase C signaling pathway. J Biol Chem. 1999; 274(46):33057-63. 
83. Morere JF, Des Guetz G, Mourad J, Levy BI, Breau J. Mechanism of bevacizumab-induced arterial hypertension: Relation with skin capillary rarefaction in patients treated for metastatic colorectal cancer. J Clin Oncol. (Meeting Abstracts) 2007;25(18 suppl):3557.

84. Mourad JJ, des Guetz G, Debbabi H, Levy BI. Blood pressure rise following angiogenesis inhibition by bevacizumab. A crucial role for microcirculation. Ann Oncol. 2008;19(5):927-34.

85. Zhu X, Wu S, Dahut WL, Parikh CR. Risks of proteinuria and hypertension with bevacizumab, an antibody against vascular endothelial growth factor: systematic review and meta-analysis. Am J Kidney Dis. 2007;49(2):186-93.

86. Kabbinavar F, Hurwitz HI, Fehrenbacher L, et al. Phase II, randomized trial comparing bevacizumab plus fluorouracil (FU)/leucovorin (LV) with FU/LV alone in patients with metastatic colorectal cancer. $J$ Clin Oncol. 2003;21(1):60-5.

87. Genentech. Avastin (bevacizumab) prescribing information. 2006.

88. Gordon MS, Cunningham D. Managing patients treated with bevacizumab combination therapy. Oncology. 2005;69 Suppl 3:25-33.

89. Hurwitz H, Saini S. Bevacizumab in the treatment of metastatic colorectal cancer: safety profile and management of adverse events. Semin Oncol. 2006;33(5 Suppl 10):S26-34.

90. Hochster HS, Hart LL, Ramanathan RK, et al. Safety and efficacy of oxaliplatin/fluoropyrimidine regimens with or without bevacizumab as first-line treatment of metastatic colorectal cancer (mCRC): Final analysis of the TREE-Study. J Clin Oncol. (Meeting Abstracts) 2006;24(18 Suppl):3510.

91. Ferrara N. Role of vascular endothelial growth factor in the regulation of angiogenesis. Kidney Int. 1999;56(3):794-814.

92. Scappaticci FA, Skillings JR, Holden SN, et al. Arterial thromboembolic events in patients with metastatic carcinoma treated with chemotherapy and bevacizumab. J Natl Cancer Inst. 2007;99(16):1232-9.

93. Hambleton J, Novotny WF, Hurwitz H, et al. Bevacizumab does not increase bleeding in patients with metastatic colorectal cancer receiving concurrent anticoagulation. J Clin Oncol. (Meeting Abstracts) 2004;22 suppl 14:3528.

94. Kozloff MF, Sugrue MM, Purdie DM, et al. Safety and effectiveness of bevacizumab (BV) and chemotherapy (CT) in elderly patients (pts) with metastatic colorectal cancer (mCRC): Results from the BRiTE observational cohort study. J Clin Oncol. (Meeting Abstracts) 2008;26(15 suppl):4026.

95. Skillings JR, Johnson DH, Miller K, et al. Arterial thromboembolic events (ATEs) in a pooled analysis of 5 randomized, controlled trials (RCTs) of bevacizumab (BV) with chemotherapy. J Clin Oncol. (Meeting Abstracts) 2005;23(16 suppl):3019.

96. Flynn PJ, Sugrue MM, Purdie DM, et al. Serious bleeding events are uncommon in patients (pts) with netastatic colorectal cancer (mCRC) receiving bevacizumab (BV) as part of a first-line regimen: Results from the BRiTE Observational Cohort Study. Gastrointestinal Cancers Symposium. 2008; Abstract 346.
97. Purdie DM, Berlin JD, Flynn PJ, et al. The safety of long-term bevacizumab use: Results from the BRiTE observational cohort study (OCS). $J$ Clin Oncol. (Meeting Abstracts) 2008;26(15 suppl):4103.

98. Nissen NN, Polverini PJ, Koch AE, Volin MV, Gamelli RL, DiPietro LA. Vascular endothelial growth factor mediates angiogenic activity during the proliferative phase of wound healing. Am J Pathol. 1998;152(6):1445-52.

99. Hurwitz H, Fehrenbacher L, Cartwright T, et al. Wound healing/ bleeding in metastatic colorectal cancer patients who undergo surgery during treatment with bevacizumab. J Clin Oncol. (Meeting Abstracts) 2004;22 14 suppl:3702.

100. Scappaticci FA, Fehrenbacher L, Cartwright T, et al. Surgical wound healing complications in metastatic colorectal cancer patients treated with bevacizumab. J Surg Oncol. 2005;91(3):173-80.

101. Sugrue MM, Purdie DM, Flynn PJ, et al. Serious wound healing complications (WHCs) in patients (pts) with metastatic colorectal cancer (mCRC) receiving bevacizumab (BV) as part of a first-line regimen: Results from the BRiTE Observational Cohort Study. Gastrointestinal Cancers Symposium. 2008; Abstract No 450.

102. Sugrue MM, Purdie DM, Feng S, et al. Serious wound healing complications (sWHC) following surgery in patients (pts) with metastatic colorectal cancer ( $\mathrm{mCRC}$ ) receiving bevacizumab (BV): Results from the BRiTE observational cohort study (OCS). J Clin Oncol. (Meeting Abstracts) 2008;26(15 suppl):4105.

103. Berry S KA, Cunningham D. Lack of effect of starting bevacizumab shortly after venous access device implantation on wound healing/bleeding complications - Preliminary results from first BEAT Gastrointestinal Cancers Symposium, San Francisco; 2006. p. 208.

104. Sugrue M, Kozloff M, Hainsworth J, et al. Risk factors for gastrointestinal perforations in patients with metastatic colorectal cancer receiving bevacizumab plus chemotherapy. J Clin Oncol. (Meeting Abstracts) 2006;24(18 suppl):3535.

105. Saif MW, Elfiky A, Salem RR. Gastrointestinal perforation due to bevacizumab in colorectal cancer. Ann Surg Oncol. 2007;14(6):1860-9.

106. Badgwell BD, Camp ER, Feig B, et al. Management of bevacizumabassociated bowel perforation: a case series and review of the literature. Ann Oncol. 2008;19(3):577-82.

107. Hinchey J, Chaves C, Appignani B, et al. A reversible posterior leukoencephalopathy syndrome. N Engl J Med. 1996;334(8):494-500.

108. Garg RK. Posterior leukoencephalopathy syndrome. Postgrad Med J 2001;77(903):24-.

109. Common Terminology Criteria for Adverse Events, Version 3.0. DCTD, NCI, NIH, DHHS, 2006. Accessed July 28, 2008, at http://ctep. cancer.gov/forms/CTCAEv3.pdf. 